\title{
A comparison of long-term results after Baerveldt 250 implantation in advanced uveitic vs. other forms of glaucoma
}

\author{
Ioana Maria Cazana' ${ }^{1}$ - Daniel Böhringer ${ }^{2} \cdot$ Thomas Reinhard $^{2} \cdot$ Alexandra Anton $^{3} \cdot$ Thomas Ness $^{2} \cdot$ Jan Lübke $^{2}$
}

Received: 10 August 2021 / Revised: 16 February 2022 / Accepted: 22 February 2022 / Published online: 7 March 2022

(c) The Author(s) 2022

\begin{abstract}
Introduction Uveitic glaucoma remains challenging despite medical and surgical advancements and can potentially lead to blindness if left uncontrolled. Conservative alternatives as well as microinvasive surgeries can postpone the necessity of a highly invasive intervention. However, such procedures are still necessary to treat some refractive glaucoma cases. Since previous studies have reported excellent results following the primary implantation of glaucoma drainage devices, it was our study's aim to evaluate long-term results following a Baerveldt 250 implantation in highly complex and surgically burdened uveitic glaucoma eyes (UG) and compare these to a similar population suffering from other forms of glaucoma (OFG).

Material and methods We performed a retrospective analysis of all eyes (UG vs. OFG) following a Baerveldt 250 implant between 2013 and 2019. Efficacy parameters as well as post-operative complication data were extracted from our electronic data system for statistical analysis.

Results A total of 62 eyes were included in our study (24 UG and 38 OFG). UG baseline mean IOP was $35.04 \mathrm{mmHg}$ $( \pm 11.85 \mathrm{mmHg})$ with $3.08( \pm 1.13)$ topical agents, and OFG was $32.63 \mathrm{mmHg}( \pm 7.74 \mathrm{mmHg})$ with $2.68( \pm 1.28)$ topical agents. A majority of eyes also required systemic acetazolamide (UG: 79\% OFG: 87\%) and had undergone at least one glaucoma-related operation prior to the Baerveldt 250 implant ((UG: $1.21( \pm 0.66))$, OFG: $1.74( \pm 1.33))$. At the median follow-up period (UG 592, OFG 764 days), 52.5\%/32.5\% of UG/OFG cases showed qualified success (IOP below $21 \mathrm{mmHg}$ with either topical or/and systemic medication), $15 \% / 30 \%$ no longer required topical medication, and $47.5 \% / 47.5 \%$ were free of acetazolamide systemically. Moreover, $75 \% / 72.5 \%$ of eyes experienced no further pressure-related surgical event. Although sight-threatening complications such as corneal and macular edema were reported in both groups, most either maintained or improved their visual acuity at the last follow-up $(58.33 \% / 57.89 \%)$.

Conclusion The Baerveldt 250 implant is shown to be both effective and safe for advanced glaucoma cases in uveitis and other forms. No further glaucoma-related surgery is required in the majority of eyes in either group within a follow-up period of almost 2 years. Despite sight-threatening complications such as macular and corneal edema, visual acuity can be either maintained or improved in most eyes.
\end{abstract}

Keywords Baerveldt implant $\cdot$ Secondary inflammatory glaucoma $\cdot$ Uveitic glaucoma $\cdot$ Shunts $\cdot$ Drainage devices

The study was carried out at the Eye Center, University of Freiburg.

Ioana Maria Cazana

ioana-maria.cazana@usz.ch

1 Department of Ophthalmology, University Hospital of Zurich, Frauenklinikstrasse 24, 8091 Zurich, Switzerland

2 Eye Center, Medical Center, University of Freiburg, Faculty of Medicine, Freiburg, Germany

3 ADMEDICO Eye Center, Olten, Switzerland 


\section{Key messages:}

- The Baerveldt implant is shownto be both safe and effective as a first line surgical therapy in uveitic glaucoma cohorts

- The Baerveldt 250 implant is also shown to be safe and effective for advanced glaucoma cases (uveitic and others forms alike) following failed medical and surgical therapy

- No further glaucoma-related surgery is required in the majority of advanced glaucoma cases following a Baerveldt 250 implant

\section{Introduction}

With a $10-20 \%$ prevalence, glaucoma is a common and severe complication of uveitis [1,2]. Despite ongoing advancements, its management remains a complex challenge, which requires addressing both the underlying condition and an elevated intraocular pressure (IOP) simultaneously. A reduced outflow capacity after recurrent inflammation coupled by a hypertensive response to corticosteroids is responsible for the persistence of an elevated IOP despite intensive medical therapy [3-6]. Furthermore, surgical interventions are necessary in up to $35 \%$ of adults and $59 \%$ of children due to medical treatment failure [7].

A myriad of surgical options with comparable efficacies are currently available [8-14]. However, chronic inflammation can compromise the long-term outcome following both trabeculectomy ab externo and interno, and cycloablative techniques remain a last resort because of their irreversible nature [8-14]. Since a poorly controlled glaucoma may lead to blindness, urgent and aggressive forms of treatment are necessary. Glaucoma drainage devices (GDD) offer an efficient and safe alternative for combating refractory hypertension due to uveitis [9]. Specifically, the Baerveldt implant (BI) is shown to be of significant relevance in resolving such cases [15-17]. Long-term performance reports are relatively scarce. Additionally, to our knowledge, none includes highly diseased and surgically burdened study populations following a small Baerverldt 250 implant (BI-250). Filling this gap, we evaluate the long-term efficiency and safety following BI-250 in complex eyes suffering from glaucoma due to uveitis (UG) vs. other forms (OFG).

\section{Material and methods}

\section{Retrospective data}

This single-center retrospective study was performed at the Eye Center of the Medical Center, University of
Freiburg. Each tenant of the Declaration of Helsinki was upheld. Following ethics committee approval (vote no. 619/19), data was acquired based on our electronic patient management system. All patients that underwent a BI-250 between 2013 and 2019 were identified. Their last pre-operative and all follow-up medical records were reviewed, and relevant information was entered in our database. From the last pre-operative examination, age, gender, visual acuity, type of uveitis, IOP, use of medication (topical and systemic), pre-BI-250 surgeries, lens status, and presence of macular and corneal edema were extracted. At follow-up, data included as follows: visual acuity, IOP, use of medication (topical and systemic), postBI-250 surgeries, presence of macular and corneal edema/ transplantation rates, and surgical complications.

Our hospital routinely uses Goldmann applanation tonometry (Haag-Streit, Köniz, Switzerland) to measure IOP in glaucoma patients. The device is calibrated according to the manufacturer's recommendations. A complete success was defined as an IOP below $21 \mathrm{mmHg}$ without either topical or systemic medication, while a qualified success was defined as an IOP below $21 \mathrm{mmHg}$ with either topical and/ or systemic medication. Hypotony was considered as an IOP below $5 \mathrm{mmHg}$. Post-operative IOP spikes are common in the first month due to tube occlusion. Therefore, IOP was reported starting in the second post-operative month.

The total number of IOP lowering agents resulted from the sum of each category of topical drugs (beta-blockers, prostaglandin-analogues, carbonic anhydrase inhibitors, alpha2-agonists). Agents present in fixed combination eye drops were regarded individually and oral acetazolamide was considered separately.

Cases showing ophthalmoscopically ambiguous macular findings despite a stable visual acuity and those with an unexplained worsened visual acuity received further testing via optical coherence tomography (OCT) using a Spectralis OCT (Heidelberg Engineering, Dossenheim, Germany). Treatment proceeded according to cause. The presence of corneal edema drastically impacts post-operative visual outcomes and was therefore evaluated through slit lamp microscopy and endothelial cell count. Furthermore, post-BI-250 
corneal transplantations were tabulated for native and grafted eyes of both groups. Lastly, regardless of cause, we considered any visual acuity change of $0.2 \log$ MAR ( 2 lines) to be significant.

\section{Surgical procedure}

A total of four surgeons performed all Baerveldt implantations following the same surgical procedure. After an initial limbus-based conjunctival incision in the inferonasal quadrant, the BI, BG103-250 (62) (Abbott Medical Optics Inc., Santa Ana, CA, USA) was secured with its wings beneath the inferior and medial rectus muscles and its anterior edge $11 \mathrm{~mm}$ posterior to the limbus, using two dafilon $6-0$ sutures. Patency was ensured through irrigation, and tube occlusion was performed using a vicryl rapide 6-0 suture in order to prevent post-operative hypotony. After the tube was individually shortened in order for it to lay comfortably in the anterior chamber avoiding pupil-obstruction, a scleral flap was prepared and the tube was then inserted in the anterior chamber using a 23-gauge needle for tracking. The tube was attached to the globe using nylon 10-0 sutures and the scleral flap was secured and conjunctiva closed using vicryl 7-0 sutures. At the conclusion of the procedure, an intracameral steroid injection was administered via a paracentesis.

\section{Statistical analysis}

All statistical analyses were performed using R statistical software [18]. Differences in patient's baseline characteristics were analyzed using either the independent $t$ test for continuous variables or the chi-square test for categorical variables. $P$ values below 0.05 were considered statistically significant. Next, Kaplan-Meier curves were employed to provide a visualization of post-operative outcomes between both groups, and the results at the median follow-up time were extrapolated (UG vs. OFG).

\section{Results}

\section{Baseline characteristics}

A total of 62 eyes were included in our study (24 UG and 38 OFG). Of the 24 UG eyes, 54\% presented with anterior-, $29 \%$ with intermediate-, and $17 \%$ with pan-uveitis. Both groups comprised of relatively young patients, with a higher female representation in the UG group. The mean IOP was $35.04 \mathrm{mmHg}( \pm 11.85 \mathrm{mmHg})$ with $3.08( \pm 1.13)$ topical agents in the UG group, $32.63 \mathrm{mmHg}( \pm 7.74 \mathrm{mmHg})$ with $2.68( \pm 1.28)$ in the OFG group. $79 \% / 87 \%$ of eyes respectively followed a systemic acetazolamide regimen before the operation. On average, patients underwent 1 to 2 glaucoma operations before receiving a BI-250 ((UG: $1.21( \pm 0.66))$, OFG: $1.74( \pm 1.33))$. Further baseline characteristics can be found in Table 1.

\section{Post-operative results}

The median follow-up period was 592/764 days for patients in the UG/OFG cohort. At this time, 15\%/25\% registered a complete success, and $52.5 \% / 32.5 \%$ a qualified success. $15 \%$ of the UG and $30 \%$ of the OFG eyes no longer required topical medication, and $47.5 \%$ in both were free of acetazolamide systemically. $75 \% / 72.5 \%$ of eyes experienced no further pressure-related surgical event. Almost all cases requiring further surgery underwent cyclophotocoagulation. Kaplan-Meier curves were generated for each of the aforementioned efficacy criteria (see Figs. 1, 2, 3, 4, and 5).

Uveitic eyes experienced mostly transient visual acuity decreasing events, while the OFG group suffered a more lasting damage in part due to corneal deficits necessitating corneal transplantation (penetrating keratoplasty or Descemet membrane endothelial keratoplasty, DMEK). While neither of the two corneal transplant cases in the UG group required re-transplantation following the BI-250, $31.25 \%$ of the OFG group did require re-transplantation. Of these, 4 eyes underwent penetrating keratoplasty and 1 DME keratoplasty (Figs. 6, 7, and 8). In contrast, only $4.54 \% / 13.63 \%$ of native corneas in either group respectively needed a keratoplasty (UG: 1 DMEK, OFG: 2 DMEKs and 1 penetrating keratoplasty). $57.89 \%$ of eyes in the OFG group maintained or improved their visual acuity by the last followup, as did $58.33 \%$ of eyes in the UG. No cases experienced complete loss of light perception (see Fig. 9).

Hypotony was the most common BI-250-specific complication in both groups. It affected $25 \%$ of eyes in the UG group, half of which required surgical revision. $18.42 \%$ of eyes in the OFG group experienced hypotony, which led to surgical revision in $71.43 \%$ and tube-endothelial contact in $42.86 \%$. Additionally, diplopia incidence following implantation was comparable in both groups. Table 2 summarizes all BI-250-specific post-operative complications.

\section{Discussion}

Our study offers an in-depth look at post-BI-250 results stemming from complex uveitis and non-uveitis cohorts after approximately 2 years. Considering the severity of the underlying condition, we found the BI-250 to be an efficient form of treatment for both groups, relieving $75 \%$ of uveitis and $72.5 \%$ of non-uveitis eyes from further pressure decreasing surgical events.

When used as a first line of surgical treatment, the BI has yielded positive results regardless of the underlying 
Table 1 Baseline pre-operative characteristics of the study population

\begin{tabular}{|c|c|c|c|}
\hline \multicolumn{4}{|l|}{ Baseline characteristics } \\
\hline & UG & OFG & $p$-values \\
\hline Number & 24 & 38 & \\
\hline $\operatorname{Sex}(\%)$ & & & 0.08 \\
\hline Female & 62.5 & 39.5 & \\
\hline Male & 37.5 & 60.5 & \\
\hline Age (years) & 52.79 & 51.11 & 0.93 \\
\hline Lens status & & & 0.51 \\
\hline Phakic & 3 & 2 & \\
\hline Pseudophakic & 19 & 34 & \\
\hline Aphakic & 2 & 2 & \\
\hline Keratoplasty total (\%) & 8.33 & 42.10 & 0.03 \\
\hline Penetrating keratoplasty & 1 & 13 & \\
\hline DMEK & 1 & 2 & \\
\hline DSAEK & 0 & 1 & \\
\hline Macular edema (\%) & 12.50 & 2.63 & 0.31 \\
\hline \multicolumn{4}{|l|}{ Uveitis location (\%) } \\
\hline Anterior & 54 & & \\
\hline Intermediate & 29 & & \\
\hline Pan-uveitis & 17 & & \\
\hline IOP (mmHg) & $35.04( \pm 11.85)$ & $32.63( \pm 7.74)$ & 0.58 \\
\hline Topical agents & $3.08( \pm 1.13)$ & $2.68( \pm 1.28)$ & 0.25 \\
\hline Systemic acetazolamide (\%) & $79 \%$ & $87 \%$ & 0.42 \\
\hline Visual acuity (log MAR) & $0.38( \pm 0.42)$ & $1.12( \pm 0.83)$ & $<0.001$ \\
\hline Glaucoma operations before BI 250 & $1.21( \pm 0.66)$ & $1.74( \pm 1.33)$ & 0.07 \\
\hline Traculectomy ab interno & 19 & 14 & \\
\hline Trabeuculectomy ab externo & 7 & 14 & \\
\hline Cyclophotocoagulation/cryocoagulation & 0 & 15 & \\
\hline Iridectomy & 0 & 4 & \\
\hline Molteno implant & 0 & 1 & \\
\hline Cypass implant & 0 & 2 & \\
\hline Goniotomy & 0 & 1 & \\
\hline XEN implant & 0 & 1 & \\
\hline SLT & 1 & 0 & \\
\hline Median follow-up (days) & 592 & 764 & 0.10 \\
\hline Mean uveitis duration to surgery (months) & 146.72 & & \\
\hline \multicolumn{4}{|l|}{ Other form of glaucoma (\%): } \\
\hline Congenital glaucoma & & 8 & \\
\hline Developmental glaucoma & & 3 & \\
\hline Iridocorneal endothelial syndrome & & 8 & \\
\hline Neovascular glaucoma & & 3 & \\
\hline Primary open angle glaucoma & & 26 & \\
\hline Angle closure glaucoma & & 8 & \\
\hline Pseudoexfoliative glaucoma & & 5 & \\
\hline Post-traumatic glaucoma & & 13 & \\
\hline Post-operative glaucoma & & 26 & \\
\hline
\end{tabular}

glaucoma condition. More specifically, it has shown repeated efficacy and safety in uveitis burdened eyes [9, 15, 17, 19]. Ramdas et al. provide a comparison of GDD outcomes for eyes with vs. without secondary inflammatory glaucoma [9]. Because of similar performance results for both Ahmed and Baerveldt 350 implants, they analyzed both devices as one unit [9]. A significant and similar IOP reduction was noted in both groups, with $73.7 \%$ of uveitic and $62.3 \%$ of non-uveitic eyes reaching drug-free normotension after 1 year (uveitic glaucoma: from $25.9 \pm 7.7$ to $12.7 \pm 4.4 \mathrm{mmHg}(44.9 \%$ decrease; $p<0.001)$; non-uveitic glaucoma: from $27.9 \pm 9.6$ to $13.3 \pm 4.2 \mathrm{mmHg}(42.8 \%$ decrease; $p<0.001)(p=0.729))$ [9]. Our study expands on these insights, offering an in depth analysis of BI-250 efficacy and safety for a complex study population after almost 2 years. Since our population is different to available comparisons, juxtaposing our results is highly challenging $[9,15,17,19]$. Our institution reserves the BI-250 as a "last resort" for refractory eyes after repeated 
Fig. 1 Kaplan-Meier curve displaying the complete success rate for the UG group (teal) vs. OFG group (red) (IOP $<21 \mathrm{mmHg}$ without topical medication or systemic acetazolamide). Censored cases appear as a dot

Fig. 2 Kaplan-Meier curve displaying the qualified success rate for the UG group (teal) vs. OFG group (red) (IOP $<21 \mathrm{mmHg}$ with either topical medication and/or systemic acetazolamide). Censored cases appear as a dot
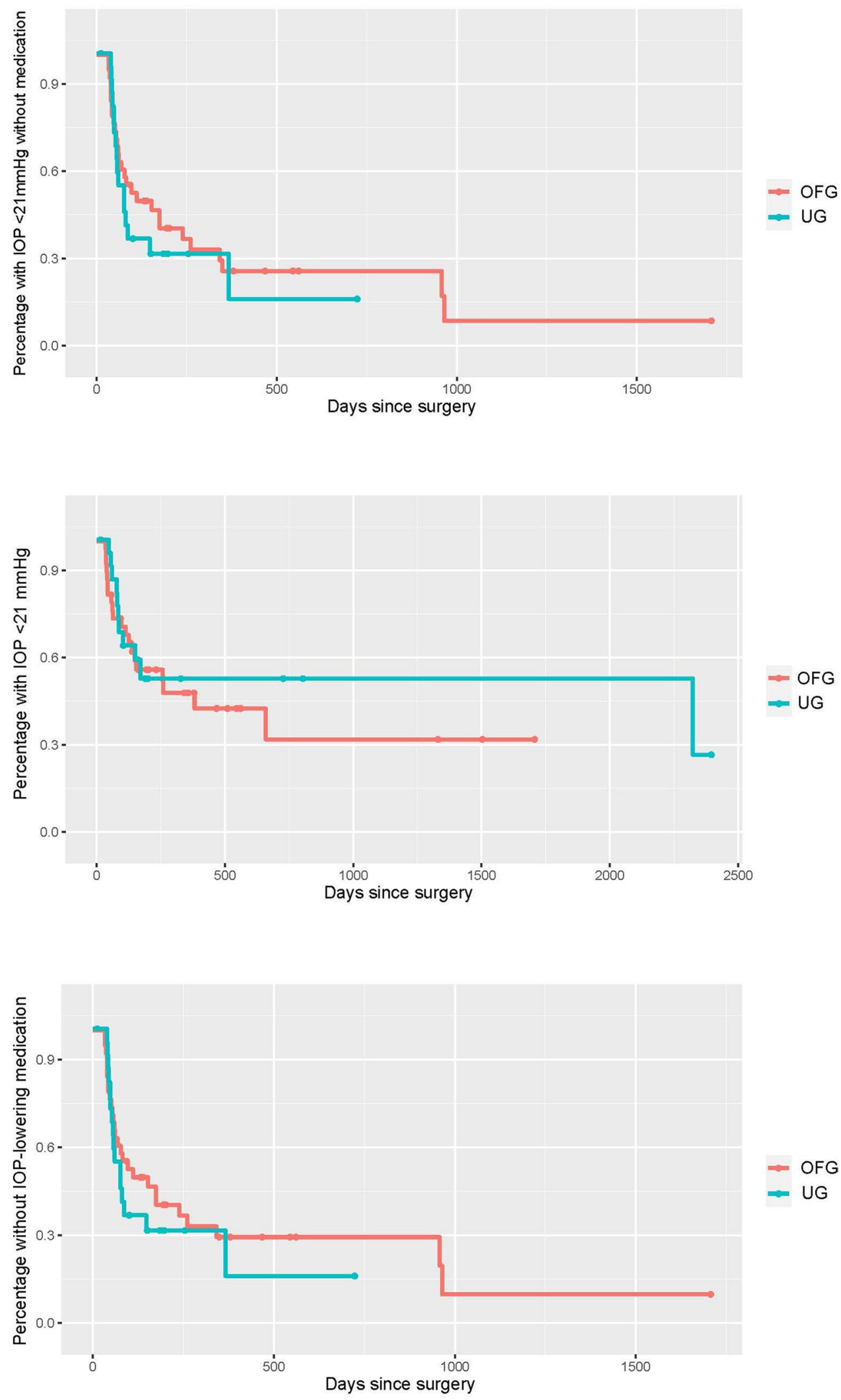

Fig. 3 Kaplan-Meier curve depicting cases post-BI-250 without topical IOP lowering medication for the UG group (teal) vs. OFG group (red). Censored cases appear as a dot 
Fig. 4 Kaplan-Meier curve depicting cases post-BI-250 without systemic acetazolamide for the UG group (teal) vs. OFG group (red). Censored cases appear as a dot

Fig. 5 Kaplan-Meier curve depicting cases post-BI-250 without further glaucomarelated surgeries for the UG group (teal) vs. OFG group (red). Censored cases appear as a dot

Fig. 6 Kaplan-Meier curve illustrating macular edema prevalence post-BI-250 for the UG group (teal) vs. OFG group (red). Censored cases appear as a dot
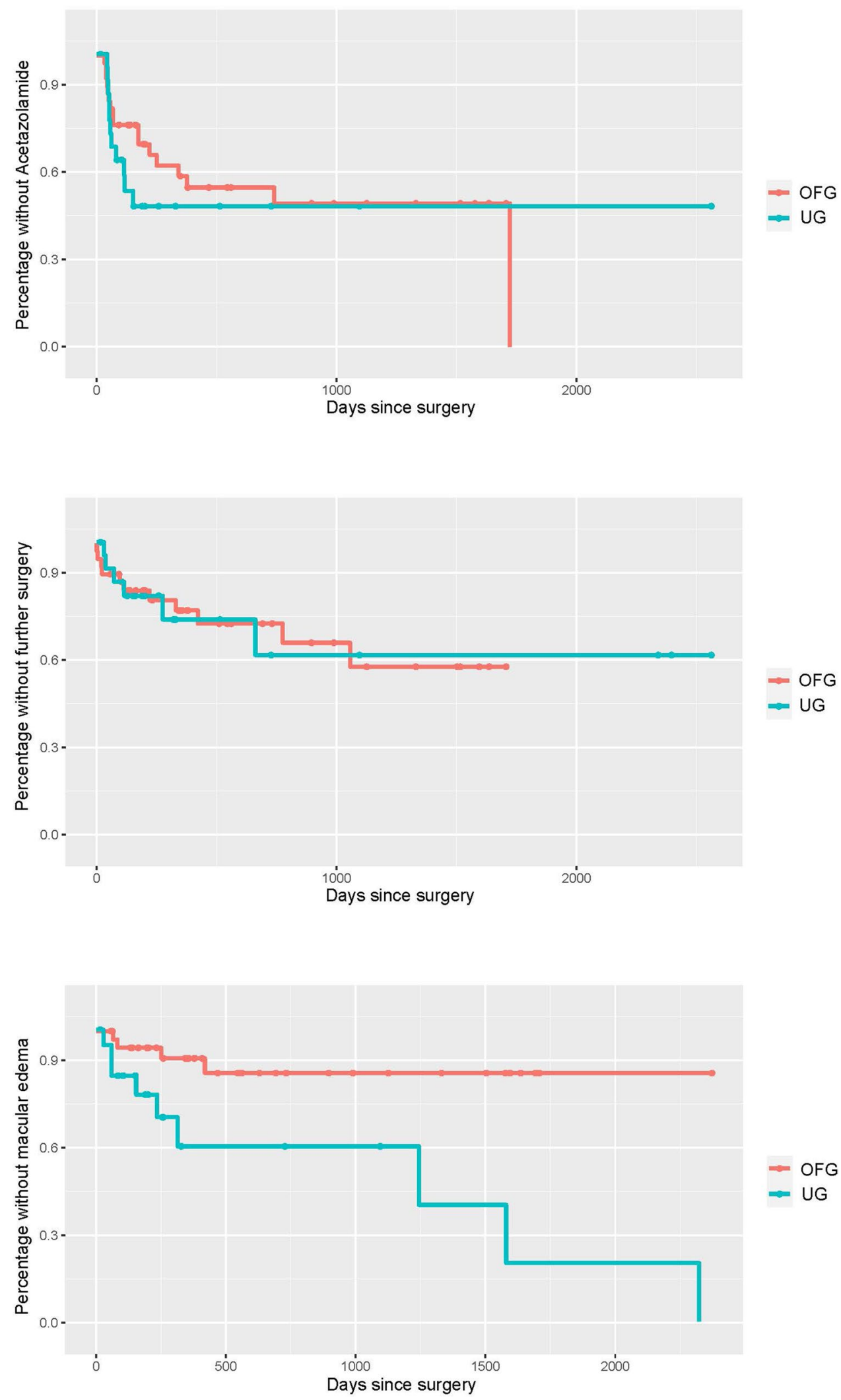
Fig. 7 Kaplan-Meier curve depicting corneal edema prevalence post-BI-250 for the UG group (teal) vs. OFG group (red). Censored cases appear as a dot

Fig. 8 Kaplan-Meier curve illustrating visual acuity stability post-BI-250 for the UG group (teal) vs. OFG group (red). Censored cases appear as a dot

Fig. 9 Visual acuity change post-BI-250 for the UG and OFG
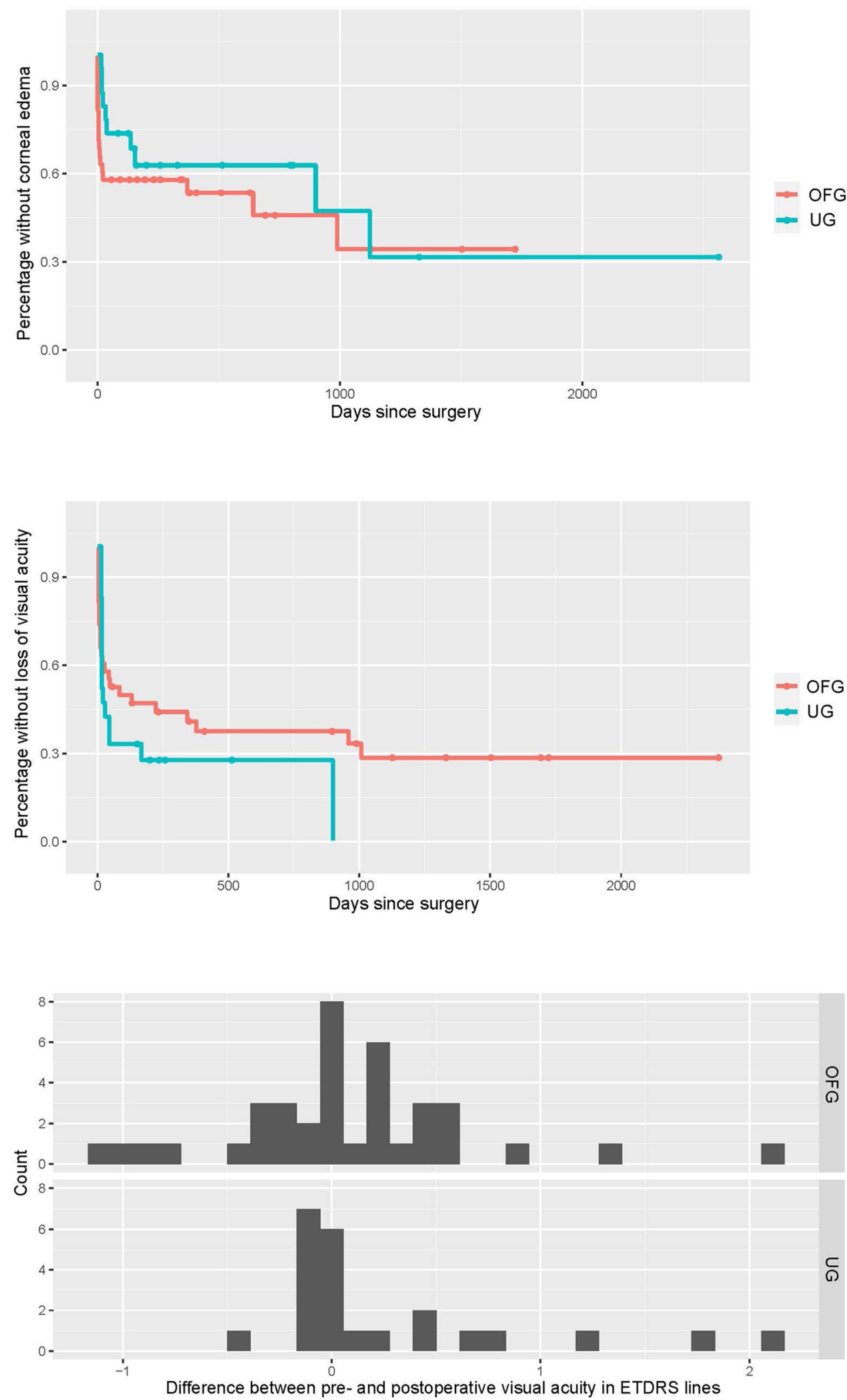

medical and surgical treatment failure. On average, each eye in both groups had undergone one to two previous pressure decreasing interventions (UG: $1.21( \pm 0.66))$, OFG: 1.74
$( \pm 1.33)$. In most cases of hypertension caused by uveitis, relative IOP reduction is primarily achieved through trabeculectomy ab interno [8]. Often and when necessary, this 
Table 2 Post-operative complications in both UG and OFG groups

Post-operative complications

\begin{tabular}{lll}
\hline & UG $(\%)$ & OFG $(\%)$ \\
\hline Hypotension $(<5 \mathrm{mmHg})$ & 25 & 18.42 \\
Revision due to hypotony & 50 & 71.43 \\
Endothelial-touch due to hypotony & 0 & 42.86 \\
Inflammation & 4.17 & 0 \\
Infection & 0 & 0 \\
Tube dislocation & 0 & 0 \\
Diplopia & 4.17 & 5.26 \\
Expulsive hemorrhage & 0 & 2.63 \\
Retinal detachment & 4.17 & 0 \\
Hypertension & 0 & 5.26 \\
\hline
\end{tabular}

is easily combined with a simultaneous cataract extraction (pseudophakia: 79.17\% UG/89.47\% OFG). In our study, $42.10 \%$ of the non-uveitic eyes also underwent some form of corneal transplantation. Conjunctival scarring and scleral deterioration that result from multiple procedures, together with a decreased risk of post-operative movement restriction resulting in double vision, led to our surgeons opting for the small $250 \mathrm{~mm}^{2} \mathrm{BI}$. This contrasts with previous reports, which mostly favor the medium $350 \mathrm{~mm}^{2} \mathrm{BI}$ for secondary inflammatory glaucoma $[9,15,17,19]$. While Britt et al. conclude that there is no additional advantage to an increased filtration area [20], we cannot be certain that a larger implant would not have altered our results.

A complete success was registered by $15 \% / 25 \%$ of eyes in the UG/OFG group, while $52.5 \% / 32.5 \%$ of UG/OFG cases experienced a qualified success post-operatively. Furthermore, $15 \%$ of the UG and $30 \%$ of the OFG eyes no longer required topical medication, $47.5 \% / 47.5 \%$ were free of acetazolamide systemically, and $75 \% / 72.5 \%$ of eyes were spared from further pressure-related interventions. At first glance, our outcomes seem to be slightly inferior to other studies reporting on inflammatory glaucoma treated with a BI. When examining 24 uveitic eyes that had undergone a BI due to refractory medical treatment, Ceballos and colleagues reported a success rate of $91.7 \%$ at 24 months, with $58.3 \%$ eyes free of antiglaucoma medications at last follow-up [15]. Furthermore, 39 patients examined by Tan et al. after 24 months reached an average IOP of $10.9 \pm 4.9 \mathrm{mmHg}$ with $1.1 \pm 1.3$ medications and $44 \%$ of eyes no longer requiring any type of pressure reducing drugs [17]. Additionally, Iverson et al. found no statistical difference between 23 eyes receiving either a $350-\mathrm{mm}^{2}$ $(n=17)$ or $250-\mathrm{mm}^{2}(n=6) \mathrm{BI}$, and the 5-year failure rate of the BI was $25 \%$ [19]. The meta-analysis conducted by Ramdas et al. then showed that $6-65 \%$ of eyes suffering from uveitic glaucoma no longer used any IOP-lowering medication after the implantation of either an Ahmed FP7 or a Baerveldt-350 drainage device (compared to 3-83\% in the glaucoma mixture studies) [9]. This emphasizes the wide spectrum of outcomes resulting from heterogeneous study populations and consequently the necessity for supplementary data. The eyes we examined generally showed a higher starting pressure and had undergone more glaucoma- and non-glaucoma-related operations. This can in part be attributed to lengthy preexisting underlying uveitic conditions (averaging 146.72 months). The severity of the pre-surgical ocular status of both groups can be deduced from the uncontrolled elevated pressure that persisted despite over $75 \%$ of eyes receiving systemic carbonic anhydrase inhibitors, which are more potent than their topical counterparts [21]. As previously mentioned, the relevance of the BI size can also not be excluded from the interpretation of our data as no previous studies have primarily reported on the $250 \mathrm{~mm}^{2} \mathrm{BI}$.

Regarding sight-threatening events, our UG group experienced more cases of decreased visual acuity post-operatively due to macular edema, while the OFG group's visual acuity drop was mostly caused by corneal edema. This is concordant with meta-analyses that show post-operative macular edema development in 2-26\% of inflammatory glaucoma vs. $2-4 \%$ in glaucoma mixture studies [9]. Our $40 \%$ prevalence is higher than previous accounts. In a study with over $70 \%$ anterior uveitis, Iverson et al. report macular edema developing in $26 \%$ of eyes following Baerveldt implantation [19]. Macular edema is less common in anterior uveitis compared to eyes suffering from intermediate-, posterior-, or pan-uveitis [22-24]. Therefore, we can attribute our increased occurrence to the inclusion of $46 \%$ intermediate- or pan-uveitis. To date, no studies have thoroughly examined the prevalence of corneal edema post-BI-250 in uveitic glaucoma. Available mixture glaucoma reports show a rate of persistent corneal edema ranging from 5.6 to $17.5 \%$ [25, 26]. Corneal endothelium deteriorates at a rate of $0.6 \%$ yearly [27]. After undergoing trabeculectomy ab externo, the rate increases to about $10 \%$ per year $[28,29]$. In contrast, no significant change is experienced after trabectome surgeries [30]. While the majority of uveitic eyes in our study had previously undergone a trabeculectomy ab interno, many of the OFG group underwent an ab externo trabeculectomy with MMC. This could partially explain the slightly higher initial corneal edema incidence in the OFG group. Additionally, $42.10 \%$ of these eyes had received some form of keratoplasty prior to the BI-250. Considering these factors, as well as the accelerated endothelial cell loss experienced after corneal transplantation [31], corneal edema susceptibility could also be due to the presence of a corneal graft. Furthermore, $31.25 \%$ of grafts necessitated a re-transplantation after BI-250 surgery, while only $13.63 \%$ of native corneas required a keratoplasty. $57.89 \%$ of eyes in the OFG group and $58.33 \%$ of eyes in the UG group either maintained or improved their visual acuity post-operatively. Tan et al. report a clinically relevant visual acuity loss in $34 \%$ of uveitic eyes [17]. However, one cannot 
directly juxtapose results since baseline visual acuities are significantly different between both studies.

Hypotony manifests in 1-38\% of eyes in heterogeneous glaucoma cohorts and in 11-36\% of secondary inflammatory glaucoma post-BI [9]. In our study, $25 \%$ of UG vs. $18.42 \%$ of OFG eyes experienced transient hypotony, which was resolved in $50 \%$ and $71.43 \%$ of cases respectively through surgical revision. The decreased pressure phase led to endothelial contact in $0 \%$ vs. $42.86 \%$ of UG/ OFG eyes. Additionally, $5.26 \%$ of eyes in the OFG group required surgical/laser interventions due to persistent hypertension caused by a fibrotic membrane and an unabsorbed tube suture. Available data show revision rates ranging from 6 to $13 \%$ in mixed glaucoma eyes and $0 \%$ in uveitic eyes [9]. Worth noting is the fact that only 4 of the 15 studies included in Ramdas et al.'s meta-analysis reported on secondary surgeries related to BIs. Moreover, the presence of mild diplopia in $4.17 \%$ of our UG group using the BI-250 is significantly below the $15 \%$ reported by Tan et al., who mainly implanted the larger $350 \mathrm{~mm}^{2}$ device [17].

\section{Conclusion}

Our data shows that the BI-250 is an effective and safe intervention for advanced glaucoma cases in uveitis as well as other forms. The majority in both our groups required no further glaucoma-related surgery within the follow-up period of almost 2 years and either maintained or improved visual acuity by the last examination. Nevertheless, sightthreatening complications including macular and corneal edema occur frequently, and grafted eyes are prone to corneal decompensation, which can lead to re-transplantation.

The advent of biologicals has provided a means of successfully controlling uveitis and its underlying condition, thus delaying the structural alterations that lead to increased ocular pressure. Moreover, the myriad of conservative alternatives and microinvasive procedures can further postpone the necessity of a highly invasive intervention. Since the BI-250 has shown high efficacy and safety in both native and surgically burdened eyes, the relevant question to be asked in further studies is not if, but when is the correct time to act drastically?

Funding Open Access funding enabled and organized by Projekt DEAL.

\section{Declarations}

Ethics approval and consent to participate The local ethics committee of the University of Freiburg approved this retrospective observational study (vote no. 619/19). Considering the retrospective nature of the study, no written consent to storing or analyzing data was necessary.

Consent for publication Not applicable.

Conflict of interest The authors declare no competing interests.

Open Access This article is licensed under a Creative Commons Attribution 4.0 International License, which permits use, sharing, adaptation, distribution and reproduction in any medium or format, as long as you give appropriate credit to the original author(s) and the source, provide a link to the Creative Commons licence, and indicate if changes were made. The images or other third party material in this article are included in the article's Creative Commons licence, unless indicated otherwise in a credit line to the material. If material is not included in the article's Creative Commons licence and your intended use is not permitted by statutory regulation or exceeds the permitted use, you will need to obtain permission directly from the copyright holder. To view a copy of this licence, visit http://creativecommons.org/licenses/by/4.0/.

\section{References}

1. Takahashi T, Ohtani S, Miyata K et al (2002) A clinical evaluation of uveitis-associated secondary glaucoma. Jpn J Ophthalmol 46:556-562. https://doi.org/10.1016/s0021-5155(02) 00549-x

2. Merayo-Lloves J, Power WJ, Rodriguez A et al (1999) Secondary glaucoma in patients with uveitis. Ophthalmol J Int Ophtalmol Int J Ophthalmol Z Augenheilkd 213:300-304. https://doi.org/ $10.1159 / 000027443$

3. Moorthy RS, Mermoud A, Baerveldt G et al (1997) Glaucoma associated with uveitis. Surv Ophthalmol 41:361-394. https://doi. org/10.1016/s0039-6257(97)00006-4

4. Peretz WL, Tomasi TB (1961) Aqueous hum or proteins in uveitis. Immunoelectrophoretic and gel diffusion studies on normal and pathological human aqueous humor. Arch Ophthalmol Chic Ill 1960 65:20-23. https://doi.org/10.1001/archopht.1961.01840 020022005

5. Toris CB, Pederson JE (1987) Aqueous humor dynamics in experimental iridocyclitis. Invest Ophthalmol Vis Sci 28:477-481

6. Armaly MF, Becker B (1965) Intraocular pressure response to topical corticosteroids. Fed Proc 24:1274-1278

7. Heinz C, Koch JM, Zurek-Imhoff B, Heiligenhaus A (2009) Prevalence of uveitic secondary glaucoma and success of nonsurgical treatment in adults and children in a tertiary referral center. Ocul Immunol Inflamm 17:243-248. https://doi.org/10.1080/09273 940902913035

8. Anton A, Heinzelmann S, Neß T et al (2015) Trabeculectomy ab interno with the Trabectome ${ }^{\circledR}$ as a therapeutic option for uveitic secondary glaucoma. Graefes Arch Clin Exp Ophthalmol Albrecht Von Graefes Arch Klin Exp Ophthalmol 253:1973-1978. https:// doi.org/10.1007/s00417-015-3102-3

9. Ramdas WD, Pals J, Rothova A, Wolfs RCW (2019) Efficacy of glaucoma drainage devices in uveitic glaucoma and a metaanalysis of the literature. Graefes Arch Clin Exp Ophthalmol 257:143-151. https://doi.org/10.1007/s00417-018-4156-9

10. Stavrou P, Murray PI (1999) Long-term follow-up of trabeculectomy without antimetabolites in patients with uveitis. Am J Ophthalmol 128:434-439. https://doi.org/10.1016/s0002-9394(99) 00185-3 
11. Carreño E, Villarón S, Portero A et al (2011) Surgical outcomes of uveitic glaucoma. J Ophthalmic Inflamm Infect 1:43-53. https:// doi.org/10.1007/s12348-010-0012-8

12. Hill RA, Nguyen QH, Baerveldt $G$ et al (1993) Trabeculectomy and Molteno implantation for glaucomas associated with uveitis. Ophthalmology 100:903-908. https://doi.org/10.1016/s01616420(93)31556-3

13. Vernon SA, Koppens JM, Menon GJ, Negi AK (2006) Diode laser cycloablation in adult glaucoma: long-term results of a standard protocol and review of current literature. Clin Exp Ophthalmol 34:411-420. https://doi.org/10.1111/j.1442-9071.2006.01241.x

14. Ataullah S, Biswas S, Artes PH et al (2002) Long term results of diode laser cycloablation in complex glaucoma using the Zeiss Visulas II system. Br J Ophthalmol 86:39-42

15. Ceballos EM, Parrish RK, Schiffman JC (2002) Outcome of Baerveldt glaucoma drainage implants for the treatment of uveitic glaucoma. Ophthalmology 109:2256-2260. https://doi.org/10. 1016/S0161-6420(02)01294-0

16. Chhabra R, Tan SZ, Au L et al (2019) Long-term outcomes and complications of Baerveldt glaucoma drainage implants in adults with glaucoma secondary to uveitis. Ocul Immunol Inflamm 27:1322-1329. https://doi.org/10.1080/09273948.2018.1517892

17. Tan AN, Cornelissen MF, Webers CAB et al (2018) Outcomes of severe uveitic glaucoma treated with Baerveldt implant: can blindness be prevented? Acta Ophthalmol (Copenh) 96:24-30. https://doi.org/10.1111/aos.13489

18. R Core Team. R: a language and environment for statistical computing. 2019. https://www.R-project.org

19. Iverson SM, Bhardwaj N, Shi W et al (2015) Surgical outcomes of inflammatory glaucoma: a comparison of trabeculectomy and glaucoma-drainage-device implantation. Jpn J Ophthalmol 59:179-186. https://doi.org/10.1007/s10384-015-0372-6

20. Britt MT, LaBree LD, Lloyd MA et al (1999) Randomized clinical trial of the $350-\mathrm{mm} 2$ versus the $500-\mathrm{mm} 2$ Baerveldt implant: longer term results: is bigger better? Ophthalmology 106:23122318. https://doi.org/10.1016/S0161-6420(99)90532-8

21. Holló G (2015) 54 - carbonic anhydrase inhibitors. In: Shaarawy TM, Sherwood MB, Hitchings RA, Crowston JG (eds) Glaucoma, 2nd edn. W.B. Saunders, pp 559-565

22. de Smet MD, Okada AA (2010) Cystoid macular edema in uveitis. Dev Ophthalmol 47:136-147. https://doi.org/10.1159/000320077
23. Fardeau C, Champion E, Massamba N, LeHoang P (2016) Uveitic macular edema. Eye 30:1277-1292. https://doi.org/10.1038/eye. 2016.115

24. Grajewski RS, Boelke AC, Adler W et al (2016) Spectral-domain optical coherence tomography findings of the macula in 500 consecutive patients with uveitis. Eye Lond Engl 30:1415-1423. https://doi.org/10.1038/eye.2016.133

25. Gedde SJ, Herndon LW, Brandt JD et al (2007) Surgical complications in the Tube Versus Trabeculectomy Study during the first year of follow-up. Am J Ophthalmol 143:23-31. https://doi.org/ 10.1016/j.ajo.2006.07.022

26. Campagnoli TR, Kim SS, Smiddy WE et al (2015) Combined pars plana vitrectomy and Baerveldt glaucoma implant placement for refractory glaucoma. Int J Ophthalmol 8:916-921. https://doi.org/ 10.3980/j.issn.2222-3959.2015.05.11

27. Bourne WM, Nelson LR, Hodge DO (1997) Central corneal endothelial cell changes over a ten-year period. Invest Ophthalmol Vis Sci 38:779-782

28. Higashide T, Nishino T, Sakaguchi K et al (2019) Determinants of corneal endothelial cell loss after trabeculectomy with mitomycin C. J Glaucoma 28:61-67. https://doi.org/10.1097/IJG.0000000000 001108

29. Storr-Paulsen T, Norregaard JC, Ahmed S, Storr-Paulsen A (2008) Corneal endothelial cell loss after mitomycin C-augmented trabeculectomy. J Glaucoma 17:654-657. https://doi.org/10.1097/IJG. 0b013e3181659e 56

30. Kasahara M, Shoji N, Matsumura K (2019) The influence of trabectome surgery on corneal endothelial cells. J Glaucoma 28:150 153. https://doi.org/10.1097/IJG.0000000000001128

31. Reinhard T, Böhringer D, Sundmacher R (2001) Accelerated chronic endothelial cell loss after penetrating keratoplasty in glaucoma eyes. J Glaucoma 10:446-451. https://doi.org/10.1097/ 00061198-200112000-00002

Publisher's note Springer Nature remains neutral with regard to jurisdictional claims in published maps and institutional affiliations. 